\title{
Geheime Netzwerke im Militär: Einführung
}

\author{
von \\ Gundula Gahlen/Daniel Marc Segesser / Carmen Winkel
}

Der militärische Widerstand im Dritten Reich hat einen prominenten Platz in der Geschichtsforschung ${ }^{1}$. Auch ist seit langem bekannt, dass insbesondere Offiziere in Geheimbünden wie zum Beispiel den Freimaurern, Rosenkreuzern oder Illuminaten von der Mitte des 18. bis weit ins 19. Jahrhundert massenhaft vertreten waren. Dies gilt sowohl für die schwedische ${ }^{2}$, englische ${ }^{3}$, französische ${ }^{4}$ oder preußische Armee ${ }^{5}$ als auch für die Kontinentalarmee des amerikanischen Unabhängigkeitskrieges ${ }^{6}$.

Die Wechselwirkungen zwischen Geheimbundorganisationen und dem Militär wurden bisher jedoch weder von der Militärgeschichtsschreibung noch der Geheimbundforschung einer eingehenden Analyse unterzogen ${ }^{7}$. Zudem bestehen deutliche Forschungsdesiderate zu geheimen Netzwerken, die innerhalb des Militärs oder initiiert durch das Militär existierten. Dies gilt für geheime Vereinigungen von Militärangehörigen ebenso wie für geheime paramilitärische Verbände und Einheiten, die vom militärischen Geheimdienst ${ }^{8}$ oder

1 Vgl. hierzu Winfried Heinemann, Plädoyer für eine Geschichte des Widerstands. In: Das ist Militärgeschichte! Probleme - Projekte - Perspektiven, hrsg. von Christian Th. Müller und Matthias Rogg, Paderborn u. a. 2013, S. 429-442. Hierin gibt Heinemann einen Überblick über die Fülle der Forschung und weist daneben mit dem Hinweis, dass die »Geschichte des militärischen Widerstands [...] keineswegs sausgeforscht« sei, auf noch ausstehende Desiderate hin.

2 Andreas Önnerfors, Freimaurerei und Offiziertum im 18. Jahrhundert. In: Militärische Eliten in der Frühen Neuzeit, hrsg. von Gundula Gahlen und Carmen Winkel, Potsdam 2010, S. 229251.

3 Robert Freke Gould, Military Lodges: The Apron and the Sword: or Freemasonry under Arms, London 1899.

4 Jean-Luc Quoy-Bodin, L’armée et la franc-maçonnerie au déclin de la monarchie sous la révolution et l'Empire, Paris 1987.

5 Karlheinz Gerlach, Die friderizianische Armee und die Freimaurer 1739-1806. In: IF Zeitschrift für Internationale Freimaurer-Forschung 7 (2005), 14, S. 9-49.

6 Steven C. Bullock, Revolutionary Brotherhood. Freemasonry and the Transformation ot the American Social Order 1730-1840, North Carolina 1996, S. 109-133; Henry J. Parker, Army Lodges during the Revolution, Boston 1884; Jacob Hugo Tatsch, Freemasonry in the Thirteen Colonies, New York 1933, S. 202-227; Sidney Kaplan, Veteran Officers and Politics in Massachusetts 1783-1787. In: William and Mary Quarterly 9 (1952), S. 29-57, hier S. 31 f; Die aktuellste Studie zu den Offizieren der Kontinentalarmee erwähnt die engen Verbindungen zwischen den Offizieren und der Freimaurerei nicht, vgl. Charles Royster, A Revolutionary People at War: The Continental Army and American Character 1775-1783, Chapel Hill, N.C. 1979.

7 Auch die Studie von Mark A. Lause stellt nicht die Beziehungen zwischen Militär und Geheimgesellschaften in den Mittelpunkt, sondern untersucht vorrangig die Rolle, die geheime Gesellschaften für die Politik im Amerikanischen Bürgerkrieg spielten, während der Krieg selbst nur am Rande erwähnt wird. Mark A. Lause, A secret society history of the Civil War, Urbana u. a. 2011.

8 Vgl. zur Geschichte der Geheimdienste Wolfgang Krieger, Geschichte der Geheimdienste. Von den Pharaonen bis zur CIA, München 2009; Geheimdienste in der Weltgeschichte. Spionage 
ähnlichen Stellen zum Zwecke der verdeckten Kriegsführung oder der Militarisierung von Staaten koordiniert wurden. Zu denken ist hier zum Beispiel an die paramilitärischen Verbände in Europa nach dem Ersten Weltkrieg' oder die Schwarze Reichswehr ${ }^{10}$. Ebenfalls zeigen sich Desiderate mit Blick auf terroristische Vereinigungen ${ }^{11}$ und Verschwörungen von Militärangehörigen ${ }^{12}$. Ausnahmen sind hier die Verschwörung des 20. Juli, einige meist im Geheimen organisierte Militärputsche ${ }^{13}$ oder der vor allem in den USA thematisierte Tatbestand der Conspiracy in den Nürnberger Prozessen ${ }^{14}$.

Der Sammelband, der auf einer Tagung in Bern vom 4. und 5. Oktober 2012 beruht, greift diese Desiderate durch einen gezielten Blick auf geheime Netz-

und verdeckte Aktionen von der Antike bis zur Gegenwart, hrsg. von dems., München 2003; Secret Intelligence in the Twentieth Century, hrsg. von Heike Bungert, Jan G. Heitmann und Michael Wala, London 2003; Peter Gudgin, Military Intelligence: A History, Phoenix Mill, U.K. 1999; Go Spy the Land: Military Intelligence in History, hrsg. von Keith Neilson und B. J. McKercher, Westport 1992.

9 War in Peace. Paramilitary Violence in Europe after the Great War, hrsg. von Robert Gerwarth und John Horne, Oxford 2012 (dt. Ausgabe: Krieg im Frieden. Paramilitärische Gewalt nach dem Ersten Weltkrieg, Göttingen 2013); dies., The Great War and Paramilitarism in Europe, 1917-23. In: Contemporary European History 19 (2010), S. 267-273; Robert Gerwarth, The Central European counter-revolution. Paramilitary violence in Germany, Austria and Hungary after the Great War. In: Past and present 200 (2008), S. 175-209; Bernhard Sauer, Vom »Mythos eines ewigen Soldatentums«. Der Feldzug deutscher Freikorps im Baltikum im Jahre 1919. In: Zeitschrift für Geschichtswissenschaft 43 (1995), S. 869-902.

10 Rüdiger Bergien, Die Soldaten der »schwarzen Reichswehr «. Motive für den Dienst in Grenzund Landesschutz zwischen 1918 und 1933. In: Das ist Militärgeschichte! (wie Anm. 1), S. 83102.

11 Vgl. zur historischen Terrorismusforschung allgemein Terrorismus, hrsg. von Martin Schulze Wessel (= Geschichte und Gesellschaft 35 (2009) Heft 3); Critical Terrorism Studies. A New Research Agenda, hrsg. von Richard Jackson u. a., London 2009; Andrew Sinclair, An Anatomy of Terror. A History of Terrorism, London 2003.

12 Vgl. z. B. Johannes Hürter, Rekruten für die »Stadtguerilla«. Die Suche der RAF nach einer personellen Basis. In: Spießer, Patrioten, Revolutionäre. Militärische Mobilisierung und gesellschaftliche Ordnung in der Neuzeit, hrsg. von Rüdiger Bergien und Ralf Pröve, Göttingen 2010, S. 305-322; vgl. auch das Themenheft Historical Social Research. Historische Sozialforschung 38 (2013), 1: Security and Conspiracy in History, $16^{\text {th }}$ to $21^{\text {st }}$ century, hrsg. von Cornel Zwierlein und Beatrice de Graaf, in dem allerdings militärische Verschwörungen keine Rolle spielen.

13 Vgl. die mittlerweile klassische Studie von Eric A. Nordlinger, Soldiers in politics: Military Coups and Governments, Englewood Cliffs, New Jersey 1977; zu einzelnen Regionen oder Einzelfällen Johannes Erger, Der Kapp-Lüttwitz-Putsch. Ein Beitrag zur deutschen Innenpolitik 1919-1920, Düsseldorf 1967; Patrick McGowan, Coups and Conflict in West Africa, 19552004. In: Armed Forces \& Society 32 (2004), S. 5-23 und 234-253; Akwasi B. Assensoh und Yvette M. Alex-Assensoh, African military history and politics. Coups and ideological incursions, 1900 - present, New York u. a. 2002; Jørgen Larsen Munck, The Kornilov Revolt: a critical examination of sources and research, Aarhus 1987 oder Bernard J. Terwiel, Thailand's Political history: From the Fall of Ayutthaya to Recent Times, Bangkok 2005, S. 261-267, zur Bedeutung des Militärputsches in Siam/Thailand im Jahr 1932; vgl. auch den Beitrag von Deniza Petrova in diesem Band.

14 Jonathan A. Bush, The Prehistory of Corporations and Conspiracy in International Criminal Law: What Nuremberg Really said. In: Columbia Law Review 109 (2009), S. 1094-1262; Telford Taylor, The Anatomy of the Nuremberg Trials: A Personal Memoir, New York 1992, S. 35-39 u. 550-554. 
werke im Militär auf ${ }^{15}$. Er geht der Frage nach Bedeutung und Einfluss geheimer Netzwerke im Militär nach und bündelt hierfür sozial- und kulturgeschichtliche Perspektiven und Fragestellungen. In verschiedenen Fallbeispielen werden die Handlungsspielräume geheimer Netzwerke innerhalb des Normensystems und der hierarchischen Strukturen des Militärs analysiert.

Eine Fokussierung auf das Militär erscheint besonders interessant, da sich Mitgliedschaften im Militär und in geheimen Netzwerken in mancher Hinsicht institutionell widersprechen, aber sich zwischen den Phänomenen Militär und geheimen Netzwerken auch deutliche Parallelen zeigen. So ist die Verbindung zwischen dem Militär und dem Begriff "geheim $\ll^{16}$ eng. Die Armee ist ein zentraler Macht- und Sicherheitsfaktor eines Staates, Informationen im militärischen Bereich sind grundsätzlich in besonderer Weise gesetzlich durch das Militärgeheimnis geschützt, da der Informationsvorsprung im militärischen Bereich sehr oft über Sieg oder Niederlage bzw. über Krieg oder Frieden den Ausschlag gab und gibt $t^{17}$. Ängste vor Unterwanderung und Verrat treten und traten in diesem Bereich besonders rasch hervor. Verbunden damit war in der zweiten Hälfte des 19. Jahrhunderts ein Ausbau der militärischen Nachrichtenund Geheimdienste, deren Aufgabe es war, im Verborgenen Informationen über Geheimnisse anderer Staaten sowie geheimer und weniger geheimer Organisationen $\mathrm{zu}$ beschaffen. Gleichzeitig sollten sie versuchen, die eigenen militärischen Geheimnisse vor dem Zugriff Dritter zu schützen, was natürlich nicht immer gelang ${ }^{18}$. Schon in der Frühen Neuzeit gehörte der militärische

15 Tagungsbericht Geheime Netzwerke im Militär vom 18. Jahrhundert bis 1945. 04.10.201205.10.2012, Bern. In: H-Soz-u-Kult, 01.02.2013, <http://www.hsozkult.de/conferencereport/ id/tagungsberichte-4626> (5. August 2015) Die Tagung wurde von der Mittelbauvereinigung der Universität Bern, dem Schweizer Nationalfonds sowie dem Arbeitskreis Militär und Gesellschaft in der Frühen Neuzeit finanziell gefördert.

16 Der Begriff »geheim« ist das Gegenstück zum Begriff »öffentlich«. Das Geheime selbst kann in unzähligen Erscheinungsformen auftreten. Alles, was einer Einzelperson oder einer Gruppe vorenthalten wird, kann zum Geheimnis werden. Vgl. hierzu Knut Knackstedt, "Geheimbund «?: Yi He Ch'üan. Ein ethnologischer Beitrag zur Neubewertung des interdisziplinär relevanten Geheimbundbegriffs am Beispiel der »Boxer « in China (1774-1900), Münster 2002, S. 10, 15-17; daneben Alois Hahn, Geheim. In: Das Geheimnis am Beginn der europäischen Moderne, hrsg. von Gisela Engel u. a., Frankfurt a. M. 2002, S. 21-42.; vgl. zu Formen des vorenthaltenen Wissens: Geheimnis und Öffentlichkeit, hrsg. von Alaida Assmann und Jan Assmann, München/Paderborn 1997; vgl. zum Begriff der Öffentlichkeit Lucian Hölscher, Öffentlichkeit. In: Geschichtliche Grundbegriffe, Bd. 4, hrsg. von Otto Brunner, Werner Conze und Reinhart Kosseleck, 3. Aufl., Stuttgart 1994, S. 413-467.

17 Vgl. hierzu Eva Horn, Der geheime Krieg. Verrat, Spionage und moderne Fiktion, Frankfurt a. M. 2007, S. 135-140; Cornel Zwierlein, Sicherheitsgeschichte. Ein neues Feld der Geschichtswissenschaften. In: Geschichte und Gesellschaft 38 (2012), S. 365-386; Verena Moritz, Militärische Nachrichtendienste vor dem Ersten Weltkrieg: Voraussetzungen und Perspektiven einer Geschichte wechselseitiger Wahrnehmungen und Einschätzungen am Beispiel Österreich-Ungarns und des Zarenreichs. In: Frontwechsel: Österreich-Ungarns »Grosser Krieg« im Vergleich, hrsg. von Wolfram Dornik, Julia Walleczek-Fritz und Stefan Wedrac, Wien 2014, S. 399-420. Programmatisch dazu auch Eckart Conze, Sicherheit als Kultur. Überlegungen zu einer »modernen Politikgeschichte« der Bundesrepublik Deutschland. In: Vierteljahrshefte für Zeitgeschichte 53 (2005), S. 352-380.

18 Krieger, Geschichte der Geheimdienste (wie Anm. 8), S. 14; Moritz, Militärische Nachrichtendienste (wie Anm. 17), S. 400, 402. Interessante detaillierte Studien dazu gibt es zur Habsbur- 
Bereich, genauso wie der gesamte politische Bereich zu den Arcana Imperii des Fürsten ${ }^{19}$. Und nach den Revolutionen von 1789, 1831 sowie 1848 und der damit einhergehenden stärkeren Gewichtung rechtlicher Regeln - meist in Form von Verfassungen - wurde der Schutz des Geheimnisses beim Militär im 19. und 20. Jahrhundert dann einer speziellen Institution übertragen, der Militärjustiz. Ihre Jurisdiktion war wesentlich weiter gefasst als diejenige der Ziviljustiz, deren Kompetenzen durch die jeweilige Verfassung eingeschränkt wurden ${ }^{20}$.

Das besondere Spannungsverhältnis, welches zwischen dem Militär und geheimen Netzwerken besteht und eine Analyse reizvoll macht, beruht zudem darauf, dass das Militär ein gesellschaftliches Subsystem darstellt, welches durch eigene Normen, Rituale, Symbole sowie sprachliche und vestimentäre Codes eine spezifische »militärische Kultur « bildet. Disziplin, eine hierarchische Rang- und Befehlsstruktur sowie ein eigenes Berufsethos prägen das Militär als Organisation bis heute ${ }^{21}$. Geheime Netzwerke, in denen Militärangehörige agieren, fügen sich nicht deckungsgleich in das Subsystem Militär ein und verfügen vielfach über abweichende Direktiven, Rangordnungen, Verhaltenscodices und Zeichen. Sie haben damit das Potential, die innere Kohärenz im Militär zu schwächen und die Loyalität zu Armee und Regierung zu schmälern. Entsprechend häufig erregen sie den Verdacht, dass sie gegen dieses System oder den Staat gerichtet $\operatorname{sind}^{22}$.

Der Sammelband behandelt die Zeit vom 18. Jahrhundert bis zum Ende des Zweiten Weltkriegs. Zwar haben geheime Vereinigungen von Menschen zu allen Zeiten und in den meisten Gesellschaften bestanden und »gebören [...] [damit] zum Urgestein der Menschbeitsgeschichte «"23, doch ergibt sich die Begrenzung auf die Zeit seit dem 18. Jahrhundert aus dem Faktum, dass sich der moderne Staat und seine Kultur zusammen mit Geheimgesellschaften und

germonarchie und der Republik Österreich von Verena Moritz, Schattenwelten: Die Aktivitäten der militärischen Geheimdienste während des Ersten Weltkrieges. In: Jubel\&Elend: Leben mit dem Grossen Krieg, red. von Peter Fritz und Christian Rapp, Schallaburg 2014, S. 350-355, dies. und Hannes Leidinger, Oberst Redl: Der Spionagefall, der Skandal, die Fakten, Wien 2013 sowie dies. und Gerhard Jagschitz, Im Zentrum der Macht: Die vielen Gesichter des Geheimdienstchefs Maximilian Ronge, Wien 2007. Ebenfalls mit der Problematik geheimer und geheimdienstlicher Tätigkeit im Vorfeld des Ersten Weltkrieges beschäftigt sich Christopher Clark, The Sleepwalkers: How Europe Went to War in 1914, London 2012, S. xxiii, 38-42 u. 94-99.

19 Vgl. hierzu allgemein Anja Victorine Hartmann, Arcana Imperii und Theatrum Mundi. Überlegungen zur Bedeutung des Geheimnisses in der Frühen Neuzeit. In: Geschichte in Wissenschaft und Unterricht 53 (2002), S. 434-443; Michael Stolleis, Arcana imperii und Ratio status. Bemerkungen zur politischen Theorie des frühen 17. Jahrhunderts, Göttingen 1980.

20 Peter Judson Richards, Extraordinary justice. Military tribunals in historical and international context, New York u. a. 2007; Gary D. Solis, The law of armed conflict. International humanitarian law in war, New York 2013.

21 Ulrich vom Hagen, Maren Tomforde, Militärische Organisationskultur. In: Militärsoziologie - Eine Einführung, hrsg. von Nina Leonhard und Ines-Jacqueline Werkner, Wiesbaden 2005, S. $176-195$.

22 Vgl. etwa den Beitrag von Gundula Gahlen zur Angst vor geheimen Gesellschaften im bayerischen Militär in diesem Band.

23 Gerd-Klaus Kaltenbrunner, Vorwort. In: Geheimgesellschaften und der Mythos der Weltverschwörung, hrsg. von dems., Freiburg i. Br. u. a. 1987, S. 7-23, hier S. 7. 
verschwörerischen Gruppierungen entwickelten. Das 18. Jahrhundert wurde schon von Zeitgenossen als das goldene Zeitalter der arkanen Sozietäten angesehen. So schrieb Adolph Freiherr von Knigge (1752-1796) im Jahre 1788: "Man wird beut zu Tage in allen Ständen wenig Menschen antreffen, die nicht von Wißbegierde, Thätigkeitstrieb, Geselligkeit oder Vorwitz geleitet, wenigstens eine Zeitlang Mitglieder einer solchen gebeimen Verbrüderung gewesen wären ${ }^{24}$ ". Und der deutsche Philosoph und Soziologe Georg Simmel sah in seiner Soziologie des Geheimnisses das 18. Jahrhundert als besonders »prädestiniert « "ür das Aufwachsen gebeimer Gesellschaften « an, da damals die aufkeimenden liberalen Ideen auf staatliche Widerstände gestoßen seien, die in den geheimen Gesellschaften überwunden werden konnten ${ }^{25}$. Reinhart Koselleck vertrat schließlich in seiner klassischen Studie »Kritik und Krise. Ein Beitrag zur Pathogenese der bürgerlichen Welt « die These, dass »die Aufspaltung des Menschen in das Private und das Etatistische [...] für die Genese des Gebeimnisses « konstitutiv sei. Die Dialektik von Aufklärung und Geheimhaltung sei dabei »bereits an der Wurzel des absolutistischen Staates angelegt ${ }^{26}$.

Mit dem Ende des Zweiten Weltkrieges endet der Sammelband. Auch wenn es falsch wäre, von einer globalen Zäsur ${ }^{27}$ (oder gar einer Stunde Null ${ }^{28}$ ) im Jahr $1945 \mathrm{zu}$ sprechen, so waren die Jahre am Ende des Zweiten Weltkrieges mit den Prozessen von Nürnberg und Tokio, der Machtübernahme der Kommunisten in China, der neuen Rolle des Tenno in Japan, der Spaltung Europas und Koreas im Zeichen des Kalten Krieges, der »endgültigen « Lösung der Dominions Australien und Neuseeland von Großbritannien und deren Hinwendung zu den USA (ANZUS) globalgeschichtlich doch von Bedeutung ${ }^{29}$. Auch für

${ }_{24}$ Adolph Freiherr von Knigge: Über den Umgang mit Menschen, hrsg. von Gerd Ueding, Frankfurt a. M. und Leipzig 2001, S. 391.

25 Er schrieb: »Deshalb sind Zeiten, in denen sich neue Lebensinhalte unter dem Widerstande der bestehenden Gewalten aufarbeiten, für das Aufwachsen geheimer Gesellschaften prädestiniert, wie etwa das 18. Jabrhundert«. Georg Simmel, Soziologie. Untersuchungen über die Formen der Vergesellschaftung, Berlin 1908, bes. S. 256-304: Kapitel V: Das Geheimnis und die geheime Gesellschaft, hier S. 284. Bereits zwei Jahre früher hatte er in einem englischsprachigen Aufsatz - Georg Simmel, The Sociology of Secrecy and of the Secret Societies. In: American Journal of Sociology 11 (1906), S. 441-498 - das Geheimnis als eine »universal sociological technique« zur Vermittlung von Wissen bezeichnet, die nicht einfach als Verschwörung bezeichnet werden dürfe.

26 Reinhart Koselleck, Kritik und Krise. Eine Studie zur Pathogenese der bürgerlichen Welt, Frankfurt a. M. 1973, S. 29.

27 Zur generellen Problematik von Zäsuren siehe Martin Sabrow, Zäsuren in der Zeitgeschichte. In: Version: 1.0. In: Docupedia-Zeitgeschichte, 3. 6.2013, einsehbar unter: <http://docupedia. de/zg/Zaesuren> (14. Juli 2015).

28 Siehe dazu die Rede von Bundespräsident Richard von Weizäcker vom 8. Mai 1985 im Plenarsaal des Deutschen Bundestages zum 40. Jahrestag der Beendigung des Krieges und der nationalsozialistischen Gewaltherrschaft, in welcher er festhielt: »Es gab keine "Stunde Null«, aber wir hatten die Chance zu einem Neubeginn. «Die Rede ist vollständig abgedruckt unter <http:// www.bundespraesident.de/SharedDocs/Reden/DE/Richard-von-Weizsaecker/Reden/1985/05/19850508_Rede.html> (5. August 2015).

29 Vgl. leider ohne explizite Thematisierung der Bedeutung der Jahre am Ende des Zweiten Weltkrieges für die Epochenbildung Neueste Zeit, hrsg. von Andreas Wirsching, München 2006, S. 89-172. Gespannt erwartet wird in diesem Zusammenhang die Publikation der Ergebnisse der Konferenz »Epochenbrüche im >kurzen< 20. Jahrhundert« am Ludwig Boltzmann Institut für Kriegsfolgen-Forschung in Graz vom 15./16. November 2012. Siehe dazu: <http://cluster- 
geheime Netzwerke, Geheimorganisationen und Nachrichten- beziehungsweise Geheimdienste änderte sich mit dem Ende des Zweiten Weltkrieges etliches, denn viele Dinge, die bisher geheim waren, traten nun an die Öffentlichkeit oder wurden dahin gezerrt - so beispielsweise in den internationalen wie nationalen Kriegsverbrecherprozessen. Umgekehrt mussten sich viele ehemals führende Nationalsozialisten, um zu überleben, in eine meist geheime innere oder äußere Emigration begeben ${ }^{30}$. Auch für die Geheimdienste gab es mit dem Beginn des Kalten Krieges erhebliche Veränderungen, dies einerseits mit Blick auf ihre Ausrichtung und andererseits hinsichtlich ihrer Organisation. Die Arbeit ging aber selbstverständlich weiter, wie das Beispiel des damals bereits pensionierten letzten Spionagechefs der Habsburgermonarchie Maximilian Ronge zeigt ${ }^{31}$.

Für die vorliegende Publikation wurde der Begriff »geheimes Netzwerk « gewählt, weil darunter andere Termini wie Geheimbund, Geheimgesellschaft, geheime Gesellschaft, Verschwörung, konspirative Vereinigung oder geheime, informelle Verbindung zusammengefasst werden können ${ }^{32}$. Zudem wird ein Anschluss an die Begrifflichkeit der historischen sozialen Netzwerkanalyse möglich. In dieser wird davon ausgegangen, dass soziale Netzwerke »die Bausteine der sozialen Welt [bilden], nicht Einzelindividuen odersoziale Gruppen « ${ }^{33}$. Unter dem Begriff des Netzwerkes wird hier ein auf Vertrauen basiertes Austauschsystem zwischen Akteuren verstanden, die sich für einen gewissen Zeitraum zur Beförderung bestimmter Absichten und Ziele zusammenschließen, und innerhalb dessen »Werte nach bestimmten Regeln zirkulieren « ${ }^{34}$.

geschichte.lbg.ac.at/sites/files/clustergeschichte/programm_cluster_epochenbrueche.pdf> (14. Juli 2015).

30 Beispielhaft sind dazu die Ausführungen von Bettina Stangneth, Eichmann vor Jerusalem: Das unbehelligte Leben eines Massenmörders, Zürich 2011 zum Fall von Adolf Eichmann, der nach dem Ende des Krieges Mühe damit hatte, nicht mehr im Mittelpunkt zu stehen und im Verborgenen leben zu müssen. Nicht zuletzt deshalb gelang es dem israelischen Geheimdienst, ihn nach seiner Entführung 1960 wieder ganz an das Licht der Öffentlichkeit zu zerren.

31 Krieger, Geschichte der Geheimdienste (wie Anm. 8), S. 250-290; zu Ronge siehe Moritz/Leidinger/Jagschitz, Im Zentrum der Macht (wie Anm. 18), S. 307-357.

32 Im 2013 erschienenen, zeitlich übergreifenden kulturgeschichtlichen Sammelband von Jost Hermand und Sabine Mödersheim wurde hingegen der Begriff »Geheimgesellschaften« als Titel gewählt: Deutsche Geheimgesellschaften. Von der Frühen Neuzeit bis zur Gegenwart, hrsg. von Jost Hermand und Sabine Mödersheim, Köln 2013. Dies führt z. B. dazu, dass auch die 2011 aufgedeckte rechtsextreme terroristische Vereinigung Nationalsozialistischer Untergrund (NSU), die in diesem Sammelband behandelt wird, unter diesen Begriff subsumiert wird, was aufgrund der heutigen Sprachgewohnheiten zumindest erklärungsbedürftig ist. Jost Hermand, Die »Döner-Morde«. Der Nationalsozialistische Untergrund. In: Ebd., S. 181-190. Vgl. auch die diesbezügliche Kritik von Frank Jacob, Rezension zu: Deutsche Geheimgesellschaften. Von der Frühen Neuzeit bis zur Gegenwart, hrsg. von Jost Hermand und Sabine Mödersheim, Köln 2013. In: H-Soz-u-Kult, 18.12.2013, <http://www.hsozkult.de/publicationreview/id/rezbuecher-20550> (5. August 2015).

33 Wolfgang Neurath und Lothar Krempel, Geschichtswissenschaft und Netzwerkanalyse: Potenziale und Beispiele. In: Transnationale Netzwerke. Historische Erkundungen zu Ideen, Praktiken, Individuen und Organisationen, hrsg. von Berthold Unfried, Jürgen Mittag und Marcel van der Linden, Wien 2008, S. 59-81, hier S. 68.

${ }_{34}$ Ebd. Diese Definition basiert auch auf: Johannes Weyer, Soziale Netzwerke. Konzepte und Methoden der sozialwissenschaftlichen Netzwerkforschung, 2. Aufl., München 2011, S. 49. Vgl. 
Ausgehend von dieser allgemeinen Definition eines sozialen Netzwerkes soll der Begriff »geheimes Netzwerk « nun näher ausgeleuchtet werden. In diesem Zusammenhang ist es wichtig zu betonen, dass dieser nicht auf konspirativumstürzlerische Verbindungen beschränkt bleiben soll, auch wenn vom heutigen Sprachgebrauch eine solche Assoziation naheliegt. Einer von vornherein auf das Konspirative festgelegten Begrifflichkeit geheimer Netzwerke widerspricht insbesondere die Tatsache, dass sich im 18. Jahrhundert grundsätzlich systemkonforme Kräfte, wie z. B. die Gold- und Rosenkreuzer, ebenfalls geheim organisierten ${ }^{35}$. Bei anderen wie etwa bei der Freimaurerei erstreckte sich das Geheimnis allein auf die Vorgänge innerhalb der Loge und auf das Ritual ${ }^{36}$. Letztlich verstanden sich die Freimaurer nicht als Geheimgesellschaft, deren Existenz nicht öffentlich werden sollte, sondern als geschlossene Gesellschaft, zu der nicht jedermann Zutritt haben konnte und sollte ${ }^{37}$.

Zentral für die in diesem Band untersuchten Netzwerke ist, dass sie in unterschiedlichem Grad Geheimhaltungsstrategien verfolgten, was sie allerdings alle verbindet, ist ihr Anliegen etwas geheim zu halten. Für alle geheimen Netzwerke gilt, dass sie durch ihr Bestreben, ein kollektiv geteiltes Geheimnis zu wahren, definiert werden. Dieses bestimmt die Wechselbeziehungen derer, die das Geheimnis gemeinsam besitzen ${ }^{38}$. Innerhalb der geheimen Netzwerke, die sich im Einzelnen in ihrer Entstehung, Mitgliederstruktur, Gedankenwelt, Intention, Organisationsform und Verhaltensweise unterscheiden, erfüllt das Geheimnis verschiedene soziale und kommunikative Funktionen ${ }^{39}$. Dessen gemeinschaftliche Wahrung grenzt die Gruppe nach außen ab, führt zur sozialen Gemeinschaft und bindet die Mitglieder aneinander ${ }^{40}$. So entsteht ein

daneben Mark Häberlein, Netzwerkanalyse und historische Elitenforschung. Probleme, Erfahrungen und Ergebnisse am Beispiel der Reichsstadt Augsburg. In: Wissen im Netz. Botanik und Pflanzentransfer in europäischen Korrespondenznetzwerken des 18. Jahrhunderts, hrsg. von Regina Dauser u. a., Berlin 2008, S. 315-328; Christoph Boyer, Netzwerke und Geschichte: Netzwerktheorien und Geschichtswissenschaften. In: Transnationale Netzwerke (wie Anm. 33), S. 47-59.

35 Manfred Agethen, Geheimbund und Utopie. Illuminaten, Freimaurer und deutsche Spätaufklärung, München 1984, S. 34 f.

36 Christoph Hippchen, Zwischen Verschwörung und Verbot. Der Illuminatenorden im Spiegel deutscher Publizistik (1776-1800), Köln 1998, S. 6.

37 Horst Möller, Vernunft und Kritik, Deutsche Aufklärung im 17. und 18. Jahrhundert, Frankfurt a. M. 1986, S. 217; Hippchen, Zwischen Verschwörung und Verbot (wie Anm. 36), S. 6. In der Forschung hat sich dafür der Begriff der »diskreten « Gesellschaft durchgesetzt. Frank Jacob, Die Thule-Gesellschaft und die Kokuryûkai. Geheimgesellschaften im global-historischen Vergleich, Würzburg 2013, S. 29. Ähnlich exklusiv waren im spätviktorianischen Großbritannien auch die »Gentlemen's Clubs« in London. Siehe dazu: Amy Milne-Smith, London Clubland: A Cultural History of Gender and Class in late-Victorian Britain, New York 2011 und zur Bedeutung solcher Clubs für Militärs und deren Geheimnisse den Beitrag von Alaric Searle in diesem Band.

38 Simmel, Soziologie (wie Anm. 25), bes. S. 256-304: Kapitel V: Das Geheimnis und die geheime Gesellschaft, hier S. 283; Mak Lau Fong, The Sociology of Secret Societies. A Study of Chinese Secret Societies in Singapore and Peninsular Malaysia, Oxford u. a. 1981, S. 8.

39 Aleida und Jan Assmann, Zur Einführung. In: Schleier und Schwelle. Archäologie der literarischen Kommunikation, hrsg. von dens., Bd. 1: Geheimnis und Öffentlichkeit, München 1997, S. 7-17.

40 Claudia Schirrmeister, Geheimnisse. Über die Ambivalenz von Wissen und Nicht-Wissen, Wiesbaden 2004, S. 105. 
klares »Drinnen« und »Draußen« und ein geschützter Ort wird geschaffen. Zugang ist im Allgemeinen nur durch Initiation möglich, wodurch Zugehörigkeit und Statuswechsel vollzogen werden ${ }^{41}$. Innerhalb der Gruppe schafft ein Geheimnis Hierarchien, die auf tatsächlichen oder vermeintlichen Wissensdifferenzen beruhen ${ }^{42}$. Zudem organisiert und strukturiert es die Kommunikation und Handlungen innerhalb der Vereinigung ${ }^{43}$.

Bei dem Versuch, die Funktionen des Geheimnisses zu umreißen, muss schließlich auch dessen Attraktion per se betrachtet werden. Ein Teil der Anziehungskraft geheimer Netzwerke beruht auf der Atmosphäre, die durch das Geheimnis erzeugt wird. Diese bewirkt Distinktion und eine Dramatisierung der Aktivitäten des geheimen Netzwerkes ${ }^{44}$. Damit bedeutet der Beitritt dazu auch immer die Chance, sein eigenes Leben aufregender zu machen und gerade für Militärangehörige im Frieden dem langweiligen Garnisonsalltag zu entfliehen ${ }^{45}$.

Geheimnisse und Techniken der Geheimhaltung wie mysteriöse Bezeichnungen, Verkleidungen und Rituale wecken aber auch bei Außenstehenden Neugier und regen die Phantasie $\mathrm{an}^{46}$, wodurch viele geheime Netzwerke in ihren Interessen, Zielen, Inhalten und Möglichkeiten weit überschätzt werden. Projektionsphänomene, Unterstellungen und Legendenbildungen führen hier häufig dazu, dass geheime Netzwerke in der Außenperspektive ganz andere Dimensionen annehmen können als aus der Sicht der Mitglieder ${ }^{47}$. Gerade in Krisenzeiten können sich diese Übertreibungen zu Verschwörungstheorien entwickeln ${ }^{48}$.

${ }^{41}$ Marco Frenschkowski, Die Geheimbünde. Eine kulturgeschichtliche Analyse, Wiesbaden 2010, S. 34, 39.

42 Vgl. hierzu Hahn, Geheim (wie Anm. 16), S. 26.

43 Durch diese Organisation und Strukturierung wird die Kommunikation nicht verhindert, sondern gesteuert. Geheimhaltung lässt sich ganz allgemein als »Vorsicht mit Kommunikation" umschreiben. Niklas Luhmann, Geheimnis, Zeit und Ewigkeit. In: ders. und Peter Fuchs, Reden und Schweigen., Frankfurt a. M. 1989, S. 101-137, hier S. 101; Barbara Stollberg-Rilinger, Zur Einführung: Das Verschwinden des Geheimnisses. In: Das Geheimnis (wie Anm. 16), S. 229-234, hier S. 229.

44 Hierauf macht Rüdiger Bergien aufmerksam, der die Motive der Freiwilligen der »Schwarzen Reichswehr « zum Beitritt untersuchte und die durch die Atmosphäre des Konspirativen erzeugte Spannung als anziehend und notwendig beschreibt, um über den unspektakulären Alltag der »Schwarzen Reichswehr« nach 1923 hinwegzusehen. Bergien, Soldaten der »schwarzen Reichswehr« (wie Anm. 10), S. 94.

45 Ob sich deswegen von einer besonderen Affinität der Militärangehörigen zu geheimen Praktiken sprechen lässt, sei dahingestellt.

46 Für den Ku Klux Klan arbeitet dies Andreas Riffel heraus, vgl.: Ders., The Invisible Empire - der Ku Klux Klan von 1866-1871. In: Geheimgesellschaften: Kulturhistorische Sozialstudien, hrsg. von Frank Jacob, Würzburg 2013, S. 237-275.

47 Frenschkowski, Geheimbünde (wie Anm. 41), S. 18, der hier insbesondere auf die Freimaurer, Jesuiten und Opus Dei verweist.

48 Zus. Jacob, Thule-Gesellschaft (wie Anm. 37), S. 5. Vgl. allgemein zu Verschwörungstheorien: Verschwörungstheorien. Anthropologische Konstanten - historische Varianten, hrsg. von Ute Caumanns und Mathias Niendorf, Osnabrück 2001; Steve Clarke, Conspiracy Theories and Conspiracy Theorizing. In: Philosophy of the Social Science 32 (2002), S. 131-150; David Coady, Conspiracy Theories and Official Stories. In: International Journal of Applied Philosophy 17 (2003) 2, S. 199-211; Thomas Grüter, Freimaurer, Illuminaten und andere Verschwö- 
Im hier vorliegenden Sammelband interessiert die Kombination von geheimen Netzwerken und dem Militär, wobei sowohl reale als auch imaginierte geheime Netzwerke in die Betrachtung einbezogen werden. Diese Netzwerke verweisen auf individuelle Handlungsspielräume in der Institution Militär, die in unterschiedlicher Weise und zu unterschiedlichen Zwecken genutzt werden konnten. Anhand der Zwecke der geheimen Netzwerke können die folgenden fünf verschiedenen Typen von geheimen Netzwerken charakterisiert werden. Ihnen lassen sich die in diesem Band versammelten Beiträge, welche in chronologischer Reihenfolge publiziert werden, zuordnen:

Eine erste Gruppe sind die esoterischen Gebeimbünde oder Gebeimgesellschaften, deren Zweck »im hermetisch-geschlossenen Bereich des Mystischen « angesiedelt ist ${ }^{49}$. Hierunter fallen zum Beispiel die Freimaurer, Rosenkreuzer oder auch der Templerorden. Die Freimaurerei stellte dabei gewissermaßen den Prototyp dar, aus dem heraus sich andere Sozietäten entwickelten. Sie diente anderen Geheimgesellschaften mehr oder weniger als Vorbild ${ }^{50}$. Bei der Analyse von esoterischen Geheimbünden ist in unserem Zusammenhang weniger von Belang, welche esoterisch-mystischen Motive und Ziele hier verfolgt wurden, sondern vorrangig inwieweit und warum die formellen und informellen Strukturen und Kanäle der esoterischen Geheimbünde für Militärangehörige attraktiv waren und wie sie von Militärangehörigen verwendet wurden. Daneben geht es auch um die Frage, welche Wechselwirkungen sich zwischen den Geheimbünden und dem Militär entfalteten.

Allison Ramsay beleuchtet in ihrem Beitrag die Ausbreitung der Freimaurer im karibischen Raum und stellt dabei das Militär als Transmissionsriemen dieser Verbreitung in den Mittelpunkt ihrer Betrachtungen. Mit der Expansion des britischen Empires in die Karibik wurden von den dort stationierten Soldaten erste Logen gegründet. Diese Regimentslogen waren entweder mit einem festen Ort verbunden, konnten aber als » Travelling Lodges « auch dem Regiment folgen. Damit erreichte die Freimaurerei in der Karibik eine schnelle und umfassende Verbreitung. Ramsay betont nicht nur die gesellschaftliche und gesellige Funktion der Logen für die Militärangehören, sondern auch deren kulturelle Prägekraft. So definierten sich nicht wenige Soldaten bis in den Tod hinein als Mitglied der Freimaurerei und als Soldaten.

Politische Verschwörungen oder Geheimbünde, in denen Militärangehörige mitwirkten, bilden eine zweite Gruppe. Ihr Zweck bestand darin, eine gesellschafts- oder staatsverändernde Wirkung zu erzielen. Für die hiesige Blickrichtung interessiert nur in geringem Maße, welche politischen Motive und Ziele

rer. Wie Verschwörungstheorien funktionieren, Frankfurt a. M. 2008; Brian L. Keeley, Of Conspiracy Theories. In: The Journal of Philosophy 96 (1999), S. 109-26; Verschwörungstheorien. Theorie - Geschichte - Wirkung, hrsg. von Helmut Reinalter, Innsbruck 2002; Wolfgang Wippermann, Agenten des Bösen. Verschwörungstheorien von Luther bis heute, Berlin 2007.

49 Monika Neugebauer-Wölk, Esoterische Bünde und bürgerliche Gesellschaft. Entwicklungslinien zur modernen Welt im Geheimbundwesen des 18. Jahrhunderts, Göttingen 1995, S. 20.

so Hippchen, Zwischen Verschwörung und Verbot (wie Anm. 36), S. 6. 
hier zum Tragen kamen, sondern vorrangig, inwieweit die militärische Organisation genutzt wurde oder inwieweit diese gerade Militärangehörige, die als Verschwörer fungierten, behinderte.

Andreas Önnerfors untersucht in seinem Beitrag » Knights of Freedom? « die Funktion des militärischen Wallhalla-Ordens für die Formation von politischen Ideen im Offizierskorps, die sich in der so genannten »Anjala-Verschwörung « gegen die Kriegspolitik des schwedischen Königs Gustav III. manifestierten. Der Wallhalla-Orden war ein exklusiver militärischer Geheimbund, der im schwedisch-finnischen Offizierkorps von 1780 bis zum Ende des Schwedisch-Russischen Krieges (1788-1790) bestand. Anhand der Organisationsstruktur, der personellen Zusammensetzung sowie der Ideologie des militärischen Wallhalla-Ordens beleuchtet Önnerfors die soziologische Funktion des dort praktizierten »Geheimnisses «.

Deniza Petrova behandelt die Putschversuche russophiler bulgarischer Offiziere gegen den Monarchen Alexander I. von 1886 sowie gegen die Regenten im Februar 1887 und zeigt die hohe Bedeutung von Untergrundpraktiken im Militär und im Staatsleben Bulgariens auf. Sie rekonstruiert, wie die Verschwörer alte Netzwerke und Kameradschaften aus der Zeit der Kämpfe gegen die osmanische Herrschaft nutzten. Diese existierten innerhalb der bulgarischen Armee, aber auch zwischen bulgarischen und russischen Militärs und hatten seit der Unabhängigkeit des bulgarischen Staates nicht an Wirksamkeit verloren, indem sie nach wie vor Identität und Loyalität prägten sowie vertraute Praktiken und Kommunikationskanäle beinhalteten. Sie ermöglichten die Teilhabe russischer Machthaber an der Verschwörung gegen den König, wenngleich die rebellierenden bulgarischen Militärs in der konkreten Ausführung des Vorhabens relativ autonom agierten. Daneben zeigt Petrovas Analyse, dass die Putschversuche das junge bulgarische Heer in seiner Loyalität erschütterten und spalteten. Während die eine Fraktion der Offiziere ein starkes Bulgarien sich nur in Anlehnung an Russland vorstellen konnte, standen viele bulgarische Offiziere den Verschwörern ablehnend gegenüber. Letztere hatten bei ihrem Wunsch nach einer Aufrechterhaltung der herrschenden Ordnung und einer Distanz zu Russland nicht nur ordnungs- und nationalpolitische Gesichtspunkte, sondern auch Karriereaspekte im Blick, da sie eine erneute Dominanz russischer Offiziere in den Führungspositionen des bulgarischen Heeres befürchteten, wie dies bis zum Serbisch-Bulgarischen Krieg von 1885 der Fall gewesen war.

Dass eine Netzwerkanalyse im Sinne der Historischen Netzwerkforschung ein sinnvoller methodischer Ansatz sein kann, wenn behutsam bei der Datenerhebung und -deutung auch bei bruchstückhafter Quellengrundlage vorgegangen wird, zeigt der Beitrag von Linda von Keyserlingk in diesem Band. Über die Netzwerkanalyse gelingen ihr Erkenntnisse über die Handlungsspielräume der Akteure, über den Einfluss von sozialen Strukturen auf deren Handlungen, über die Verteilung von Attributen zwischen den Akteuren und die Veränderung von Räumen, in denen sie im Netzwerk agierten. Fokus ihres Beitrages ist das beziehungsweise sind die Netzwerke im Umfeld des Attentats- und Staatsstreichversuchs vom 20. Juli 1944 im Deutschen Reich. Die 
Autorin zeigt dabei minutiös auf, wie die Tatsache, dass ein Staatsstreich mit seinem Ziel, eine neue Regierung aufzubauen, möglichst viele Mitverschwörer benötigt, während bei einem Attentat aus Sicherheitsgründen der Kreis der Beteiligten möglichst gering gehalten werden sollte, erhebliche Auswirkungen auf den 20. Juli 1944 hatte. Trotz der wenigen Belege, die sich aus dem konspirativen Charakter der Tätigkeiten der Beteiligten ergaben, vermag sie zu zeigen, wie die Beteiligten rekrutiert wurden und untereinander kommunizierten. Dadurch wird deutlich, dass die »Verschwörer « des 20. Juli keine einheitliche Gruppe bildeten, sondern dass sich viele Kreise und Gruppen in Form eines dynamischen Netzwerkes überschnitten, was zu einer unterschiedlich starken Einbindung der einzelnen Personen in das Gesamtnetzwerk führte. Die differierende Zentralität kann daher, so von Keyserlingk, als eine Struktur von konzentrischen Kreisen beschrieben werden, die über ein gemeinsames Zentrum und eine Peripherie verfügten, ineinander geschachtelt waren und sich daher gegenseitig ergänzen konnten. Im Unterschied zu kommunistischen Widerstandsgruppen wies das Gesamtnetzwerk der Männer des 20. Juli keine hierarchische Struktur auf. Die Tatsache, dass allerdings gerade die für die Durchführung des Attentats und der daraufhin umzusetzenden Staatsstreichpläne zentrale Teilgruppe der Militärs viel stärker als andere einer strukturierten Hierarchie unterworfen war - speziell durch den persönlichen Eid auf den Führer -, sollte den Handlungsspielraum der Soldaten erheblich stärker begrenzen, als dies bei den Zivilisten der Fall war. Das sollte fatale Konsequenzen haben.

Eine dritte Gruppe machen funktionale gebeime Netzwerke zur Durchsetzung bestimmter militärischer bzw. politischer Interessen aus, die vor dem Gesetz oder der Öffentlichkeit geheim gehalten werden sollten und an denen unter anderem Militärangehörige beteiligt waren. Anne Simone Rous stellt in ihrem Beitrag über die »Société des antisobres « eine derartige politisch-militärische Geheimgesellschaft im frühen 18. Jahrhundert vor. Die 1728 vom preuBischen König Friedrich Wilhelm I. und dem polnischen König und sächsischen Kurfürsten August II. gegründete »Gesellschaft zur Bekämpfung der Nüchternheit « war weniger barocke Fürstenspielerei als vielmehr ein Versuch, durch einen Geheimbund auf höchster diplomatischer und militärischer Ebene politische Spannungen abzubauen. In der Gesellschaft versammelten sich neben beiden Monarchen die einflussreichsten Männer des brandenburgischen und sächsischen Hofes und zelebrierten im Rahmen der Geheimgesellschaft eine exklusive Geselligkeit, die zu einem regen Briefverkehr zwischen den beteiligten Männern führte. Der hier praktizierte und sogar festgeschriebene zwanglose Verkehr untereinander führte tatsächlich zu einer politischen Annäherung der beiden Länder. So konnten kleinere diplomatische Verstimmungen auf diesem informellen Wege schnell beigelegt werden. Das Beispiel zeigt zudem, wie eng Politik, Diplomatie und Militär in dieser Zeit auch personell verzahnt waren, da die Mehrzahl der Geheimbundmitglieder hohe militärische Dienstränge bekleidete und sich außerdem auch auf diplomatischem Parkett bewegte. Die hier vorgestellte Gesellschaft stellte also kein Netzwerk im Militär dar, sondern vielmehr einen Zusammenschluss von Männern, die einer 
exklusiven Führungselite angehörten, die sich durch den engen Verkehr mit dem Fürsten charakterisierte. Der Geheimnischarakter der Gesellschaft diente dabei weniger der Abschottung nach außen als der Stabilisierung nach innen.

Informelle Netzwerke von aktiven und/oder ehemaligen Militärs, die dazu genutzt wurden, um geheime politische, militärische oder gesellschaftliche Ziele zu verfolgen, umfassen eine vierte Gruppe. Das Verbundenheitsgefühl unter Militärangehörigen war häufig stark ausgeprägt und half, die Durchsetzung der Ziele zu unterstützen und die Geheimhaltung abzusichern. Zum einen wirkte ein spezieller militärischer Korpsgeist, durch den sich die Soldaten mit einem spezifischen Werte- und Normensystem von der Zivilgesellschaft abgrenzten, zum anderen bestanden die Bande der Kameradschaft, die im jahrelangen engen Zusammenleben oder im gemeinsamen Bestehen von Extremsituationen im Krieg gewachsen waren. Und gerade die Koalitionskriegsführung, zu denken ist hier insbesondere an die Napoleonischen Kriege wie auch an die Weltkriege, beförderte die Entwicklung von transnationalen Netzwerken unter den Soldaten, die auf der gleichen Seite gekämpft hatten. Die zu Kriegszeiten geschaffenen Verbindungen überdauerten häufig den Friedensschluss $^{51}$.

Mit einem entsprechenden, in den Quellen nicht leicht fassbaren informellen Netzwerk von aktiven wie ausgeschiedenen Generalstabsoffizieren um Joachim von Stülpnagel, Friedrich Wilhelm von Willisen und Kurt von Schleicher beschäftigt sich Jürgen Kilian. Die Gruppenmitglieder suchten, wie teilweise auch andere Generalstabsoffiziere nach der Niederlage des Ersten Weltkrieges, trotz des Vertrages von Versailles auf inoffiziellen Wegen und unter Ausnutzung ihrer persönlichen Beziehungen, für einen Wiederaufstieg Deutschlands zu einer europäischen Großmacht tätig werden zu können. Neben einer kurzen Vorstellung und Charakterisierung der Personengruppe um Stülpnagel, Willisen und Schleicher, die im Gegensatz zu anderen nicht einen sofortigen, gewaltsamen Umsturz anstrebte, sondern vielmehr einen legalen, pragmatischen Weg zu gehen suchte, legt Kilian eine Analyse ihrer Aktivitäten vor. In ihren Funktionen im Truppenamt suchten die Mitglieder gezielt wichtige Funktionen im militärischen Apparat der Weimarer Republik durch Personen ihres Vertrauens zu besetzen. Die Männer vertraten dabei die Ansicht, dass es in einem zukünftigen Krieg unabdingbar sein würde, alle Kräfte der Nation in einem Krieg zu mobilisieren ${ }^{52}$, was ein Unbehelligtbleiben der zivilen Bereiche, das der Chef des Truppenamtes Hans von Seeckt und die älteren Befehlshaber der Reichswehr noch für möglich hielt, ausschloss. Mit der Zeit kam es ob der unterschiedlichen Auffassungen zum Konflikt, der vorerst zur Versetzung Stülpnagels und anderer Offiziere führte, schließlich aber - auch gerade auf Grund der umfangreichen Vernetzung der Gruppe - mit dem Abgang Seeckts endete. Damit aber fiel ein wesentliches, konstituierendes Element für den Erhalt der Gruppe weg, was zum Zerfall des Netzwerkes führte.

51 Vgl. etwa Gerwarth, Paramilitary violence (wie Anm. 9), S. 182 f.

52 Vgl. dazu An der Schwelle zum Totalen Krieg: Die militärische Debatte über den Krieg der Zukunft 1919-1939, hrsg. von Stig Förster, Paderborn 2002. 
In seinem Beitrag unter dem Titel »Mind Britain’s Business « beschäftigt sich Alaric Searle auf der Grundlage der Definition einer Geheimgesellschaft von Georg Simmel mit faschistischen Splittergruppen und britischen Offizieren in ihrem Widerstand gegen einen Krieg zwischen Großbritannien und dem Deutschen Reich in den Jahren 1937 bis 1941. Ausgehend von der Tatsache, dass die faschistischen Splittergruppen und viele britische Offiziere der Zwischenkriegszeit eine gemeinsame ideologische Grundausrichtung (Antisemitismus, Antibolschewismus, Hochachtung vor dem Deutschen Reich und seinem Militär) teilten, kam es in den dreißiger Jahren zur Bildung verschiedener, sich vielfach überlappender Netzwerke. Diese teilten eine gemeinsame, militärisch geprägte Haltung, trafen sich an Orten, die wie die Londoner »Gentlemen's Clubs « nicht öffentlich zugänglich waren, und achteten auf Geheimhaltung. Letzteres hing nicht zuletzt damit zusammen, dass zwei wichtige Figuren in diesen Netzwerken, nämlich Generalmajor J. F. C. Fuller und Admiral Barry Domvile, aus ihrer aktiven Zeit im Militär direkte oder indirekte Kenntnisse über das Funktionieren des britischen Inlandsgeheimdienstes besaßen, die sie den übrigen Mitgliedern des Netzwerkes auch immer wieder weitergaben. Die Geheimniskrämerei der Beteiligten war mit der Zeit aber auch den Umständen geschuldet, so insbesondere dem Eintritt Großbritanniens in den Zweiten Weltkrieg, welcher aber keineswegs das Ende der Aktivitäten der faschistischen Splittergruppen und der mit ihnen verbundenen Offiziere zur Folge hatte. Ihr Einfluss war teilweise erstaunlich hoch. So wurde kurz nach dem Beginn des Krieges gar in Betracht gezogen, Fuller zum Stellvertreter des Chiefs of the Imperial General Staff, Edmund Ironside, zu machen, was schließlich, wohl nicht zuletzt auch auf Grund der engen Beziehungen Fullers zur British Union of Fascists unterblieb. Searle zeigt in seinem Beitrag deutlich auf, dass die Existenz von modernen, organisierten Geheimdiensten die Handlungsoptionen für geheime Gesellschaften und Netzwerke erschwerte, sie aber keineswegs verunmöglichte. Entsprechende Aktivitäten wurden verstärkt als potentiell gefährliche »Verschwörungen« verstanden und führten 1941 schließlich auch zur Internierung, wenngleich in den meisten Fällen nicht zu einer strafrechtlichen Verfolgung vieler Mitglieder der faschistischen Splittergruppen und der mit ihnen verbundenen Netzwerke. Sogar Admiral Domvile teilte dieses Schicksal, dies im Unterschied zum wohl von höchster Stelle protegierten Fuller. Auch wenn es zwischen den von Searle analysierten Gruppen und Netzwerken sowie den Geheimgesellschaften früherer Jahrhunderte wesentliche Unterschiede gab, so wiesen sie doch etliche ähnliche Merkmale auf und waren alle mit dem Spannungsverhältnis zwischen Geheimnis und der Notwendigkeit einer allgemeinen Bekanntheit für die Aufrechterhaltung ihrer eigenen Existenz konfrontiert.

Eine fünfte und letzte Gruppe stellen schließlich dysfunktionale gebeime Netzwerke im Militär dar, welche die Schädigung des Militärs von innen heraus oder die Aufwiegelung der Soldaten bezweckten. Dies waren Netzwerke, die insbesondere von obrigkeitlicher Seite befürchtet und streng verfolgt wurden und die häufig nur in den Köpfen der Militärführung existierten. Gleichzeitig ist zu betonen, dass gerade seit der Einführung der allgemeinen Wehr- 
pflicht das Militär durchaus für geheime, revolutionäre Vereinigungen interessant wurde, da hier Kampftechniken erlernt werden konnten und gleichzeitig ein breiter Zugriff auf junge, ungebundene Männer möglich war, die besonderes Revolutionspotential hatten.

Ein Beispiel für ein dysfunktionales geheimes Netzwerk im Militär liefert der Beitrag von Jens Boysen über die konspirative Vorbereitung des Posener Aufstands durch polnisch-preußische Soldaten im Sommer und Herbst 1918. Er verortet diesen Aufstand in die Reihe jener Nachkriegskämpfe des Ersten Weltkrieges, die als bürgerkriegsähnliche Konflikte bzw. Staatsgründungskriege von bis dahin staatenlosen Völkern ausgefochten wurden. Boysen untersucht, wie die Strukturen innerhalb der in Posen stationierten preußischen Armee die Netzwerkbildung der Verschwörer und die gegen den deutschen Staat und dessen Armee gerichteten konspirativen Vorgänge beeinflussten und begünstigten. Dabei behandelt er Fragen nach der Wirkungsmächtigkeit der militärischen Hierarchie, nach der sich wandelnden Loyalität und nach dem legalen Rahmen von effektiv illoyalen Handlungen. Den nationalpolnisch gesinnten Militärangehörigen gelang es, die mittleren und unteren Ebenen der Militärbehörden wie auch die aufgestellten Sicherungseinheiten zu infiltrieren und zu dominieren. Das Gleiche geschah mit den preußisch-deutschen öffentlichen Ämtern in der Provinz Posen 1918. Die militärischen und zivilbehördlichen Strukturen ermöglichten die Organisation der konspirativen Aktionen, zumal die meisten deutschen Militärs und Beamten nicht der polnischen Sprache mächtig waren, sodass die polnische Seite hier weitgehend offen die Kommunikationswege nutzen konnte.

Bei der Aufspaltung in diese fünf Typen ist natürlich in Rechnung zu stellen, dass zudem verschiedene Mischtypen vorkamen. Beispiele hierfür sind die Beiträge von Gundula Gablen und Vaios Kalogrias. Gahlen analysiert den Illuminateneid und seine Nachfolger in der bayerischen Armee. Der in der zweiten Hälfte des 18. Jahrhunderts in Bayern gegründete Illuminatenorden hatte mystische Züge, doch war er keine rein esoterische Geheimgesellschaft. Vielmehr wollte er durch die Unterwanderung der staatlichen Schaltstellen gesellschaftsverändernde Wirkung erzielen, sodass er gleichzeitig der Gruppe der politischen Geheimbünde zuzuordnen ist. Die Autorin untersucht das Verhältnis zwischen dem Militär und dem Illuminatenorden in Bayern, die hieraus resultierenden Unterwanderungsängste und -fantasien im bayerischen Militär sowie die rechtlichen Regelungen, welche bis zum Ende der bayerischen Armee 1919 in Kraft blieben. Offiziere waren in hoher Zahl im Illuminatenorden vertreten. Doch fügten sie sich beim Verbot des Ordens 1784 widerstandslos, traten aus und leisteten den Eid gegen geheime Gesellschaften. Insgesamt verrichtete die bayerische Armee - abgesehen von der Vertrauenskrise zum Monarchen 1848 - bis zum Ersten Weltkrieg loyal ihren Dienst. Dennoch hatte sich in Bayern die Sorge vor einer Infiltrierung des Militärapparats durch Geheimgesellschaften langfristig eingeprägt. Sie spielte erst im Kaiserreich keine Rolle mehr, als nach der bayerischen Heeresreform in Anlehnung an Preußen die Offiziere einer sehr viel umfassenderen Erziehung und Überwachung unterzogen wurden als zuvor. Dass der Eid gegen geheime 
Gesellschaften weiterhin von jedem bayerischen Offizier geschworen werden musste, lag nun an der hohen Bedeutung der katholischen Kirche in Bayern, die insbesondere die Mitgliedschaft bei den Freimaurern als unvereinbar mit dem Glaubensbekenntnis ansah.

Ein weiteres nicht eindeutig zuzuordnendes geheimes Netzwerk behandelt der Beitrag von Vaios Kalogrias über die griechische »Nationale Gesellschaft«, eine von Offizieren geleitete Geheimgesellschaft, die in den Jahren 1894-1897 von einer kleinen konspirativen Vereinigung zu einem öffentlichen machtpolitischen Faktor ersten Ranges aufstieg. Hier handelte es sich in der Anfangsphase um ein informelles militärisches Netzwerk, dessen Zweck es war, nationalpolitische Ziele durchzusetzen, die von einem Großteil der Offiziere mitgetragen wurden. Später öffnete sich die Vereinigung auch für zivile Mitglieder. Gleichzeitig zeigt sich die Nationale Gesellschaft in vielerlei Hinsicht auch als dysfunktionales Netzwerk, da sie die militärische Hierarchie unterhöhlte und die militärischen Regeln außer Kraft setzten konnte. Kalogrias analysiert die Ziele, Strukturen und Aktivitäten der Nationalen Gesellschaft sowie ihr Verhältnis zum König, der Regierung und der griechischen Zivilgesellschaft. Ein besonderes Augenmerk legt er auf die Spannungen und das Konfliktpotential innerhalb der griechischen Armee, das sich durch die Offiziere, die Mitglieder in der Nationalen Gesellschaft waren, ergab, während er gleichzeitig die Vorteile herausstellt, die das Agieren in bereits existierenden Militärstrukturen für die Geheimorganisation bewirkte. Keine andere Geheimorganisation in Griechenland konnte vergleichbares Ansehen sowie eine ähnliche Bedeutung erlangen. Und lediglich die griechische Niederlage von 1897 führte dazu, dass die Nationale Gesellschaft, welche die griechische Regierung in den Krieg gedrängt hatte, diskreditiert wurde und sich auflöste.

Die Analyse geheimer Netzwerke im Militär ist mit einer besonderen Quellenproblematik konfrontiert. In der Regel vermieden die Mitglieder von geheimen Netzwerken jede schriftliche Dokumentation ihrer Tätigkeiten, wie einige der Beiträge in diesem Band zeigen ${ }^{53}$. Folglich sind die Quellen häufig sehr lückenhaft. Viele Informationen, die für eine Netzwerkanalyse nötig wären, in der die komplexen Beziehungsstrukturen eines sozialen Netzwerks in standardisierter Form erhoben und softwaregestützt ausgewertet werden ${ }^{54}$, stehen oft

53 Vgl. die Beiträge von Vaios Kalogrias, Jürgen Kilian oder Alaric Searle. Diese Problematik, auf die auch Clark, Sleepwalkers (wie Anm. 18), S. xxiii-xxiv verweist, ist auch Krieger, Geschichte der Geheimdienste (wie Anm. 8), S. 14 und Moritz, Militärische Nachrichtendienste (wie Anm. 17), S. 403-409 bewusst. Die beiden Letzteren sind jedoch der Meinung, dass die Unterschiede $\mathrm{zu}$ anderen Bereichen der Geschichtswissenschaft nicht so groß seien, wie dies oft dargestellt werde. Einerseits sei die Quellenlage vielfach besser als angenommen, andererseits gelte es auch in diesem Themenbereich die bewährten Elemente der Quellenkritik heranzuziehen, die auch hier Ergebnisse versprechen. Dass diese Ansicht richtig ist, zeigt auch der vorliegende Band.

54 Düring, Marten und Linda Keyserlingk, Netzwerkanalyse in den Geschichtswissenschaften. Historische Netzwerkanalyse als Methode für die Erforschung von historischen Prozessen. In: Prozesse: Formen, Dynamiken, Erklärungen, hrsg. von Rainer Schützeichel und Stefan Jordan, Wiesbaden 2011. <https://www.academia.edu/449150/Netzwerkanalyse_in_den_Geschichtswissenschaften._Historische_Netzwerkanalyse_als_Methode_für_die_Erforschung_von_historischen_Prozessen> (5. August 2015); Frank Hirsch, Netzwerke in der Neueren Geschichte. In: Gläubiger, Schuldner, Arme. Netzwerke und die Rolle des Vertrauens, hrsg. von Curt W. 
nicht zur Verfügung. Zudem ist genau zu überlegen, zu welchem Zweck die vorhandenen Quellen geschrieben wurden, und ob ein Interesse existierte, dass Beziehungen und Kontakte nicht vermerkt, falsch dargestellt oder erfunden wurden ${ }^{55}$. Trotz dieser Vorbehalte gelingt es den hier versammelten Beiträgen, die ausgesuchten geheimen Netzwerke in ihren sozialen und kulturellen Kontexten detailliert zu untersuchen ${ }^{56}$. Die Analysen machen deutlich, dass ein Blick auf geheime Netzwerke im Militär dazu geeignet ist, die informellen Beziehungs- und Kontaktstrukturen im Militär zu analysieren und die Handlungsspielräume der militärischen Akteure zu vermessen ${ }^{57}$.

Hergenröder, Wiesbaden 2010, S. 133-142; Morten Reitmayer und Christian Marx, Netzwerkansätze in der Geschichtswissenschaft. In: Handbuch Netzwerkforschung, hrsg. von Christian Stegbauer und Roger Häußling, Wiesbaden 2010, S. 869-880; Boyer, Netzwerke (wie Anm. 34); Neurath und Krempel, Geschichtswissenschaft und Netzwerkanalyse (wie Anm. 34).

55 Düring und Keyserlingk, Netzwerkanalyse in den Geschichtswissenschaften (wie Anm. 54).

56 Zudem ist zu betonen, dass der Beitrag von Linda von Keyserlingk in diesem Band auf Ergebnissen einer Netzwerkanalyse basiert.

57 Bereits 1979 hat Ernst-Willi Hansen in seinem Plädoyer, das Militär soziologisch zu untersuchen, dafür geworben, diese informellen Strukturen im Militär und damit auch die Handlungsspielräume der Akteure in den Blick zu nehmen. Ernst Willi Hansen, Zur Problematik einer Sozialgeschichte des deutschen Militärs im 17. und 18. Jahrhundert. Ein Forschungsbericht. In: Zentrum für historische Forschung 6 (1979), S. 425-460, hier S. 434. 